\title{
Cure in metastatic disease
}

\author{
Hannes Kaufmann (D)
}

Received: 10 July 2018 / Accepted: 10 July 2018 / Published online: 17 July 2018

(c) Springer-Verlag GmbH Austria, part of Springer Nature 2018

One of the principles in oncology over decades was that patients diagnosed with metastatic cancer were unlikely to be cured of their disease by any means. At best, treatment with classical chemotherapy ended in extended progression-free or overall survival. Complete remissions from systemic standard chemotherapy are uncommon, and only a fraction of complete responders remain progression free for a prolonged period in most malignant diseases.

Exceptions of this paradigm according to more effective systemic and local treatment strategies are Hodgkin disease, some non-Hodgkin lymphomas, thyroid cancer and testicular germ cell tumors. Testicular germ cell tumors are among the most curable of all solid tumor malignancies, with cure being possible even in the refractory and metastatic setting. Resection of remaining viable tumor lesions after chemotherapy has become standard of care [1].

The overarching clinical question for all of us, no matter what oncological discipline we are in, is whether we can cure more patients diagnosed with metastatic disease?

There was evidence for quite a long time that patients with resectable liver only metastases suffering from colorectal cancer may achieve significant improvement in overall survival or even cure following complete resection of tumor lesions [2]. Surgery is powerful and effective, but only in those diseases that are amenable to it. Especially patients diagnosed with oligometastatic disease seem to benefit from additional local treatment strategies compared to multimetastatic cancer. Oligometastatic cancer

\section{Dr. H. Kaufmann $(\bowtie)$}

Center for Hematology and Oncology,

Kaiser-Franz-Josef-Hospital, Kundratstraße 3, 1100 Vienna, Austria

hannes.kaufmann@wienkav.at is a relatively new concept and was originally defined as five or fewer metastatic sites. It was proposed as a clinically significant state separate from the polymetastatic disease [3] and considered to be less aggressive than other metastatic phenotypes [4]. Metastasectomy should be confined to those patients groups for whom benefit has been proven, in particular of discussions according to clinical and experimental evidence supporting the role of surgery and inflammation as potential triggers for disease recurrence [5].

Recently consistent developments in the treatment of advanced cancer have led to a significant change in patient survival. During the past few years, a multidisciplinary treatment approach has become an obligatory component of the management in cancer patients.

Due to advances in available multimodality therapies and a better understanding of tumor biology and its microenvironment, survival of stage IV patients is constantly improving. The impressive therapeutic success of antibodies inhibiting immune checkpoints has extended the use of these antibodies to previously unanticipated tumor types. Results are promising and eventually there is also a potential to cure these patients without surgery. Nevertheless, the data will be not consistent, and we do not have any biomarkers to select patients who will be cured with these types of therapies and do not relapse.

Nowadays there are different possibilities to achieve complete remission and long-term survival in several types of metastatic cancer.

Definition of cure is heterogeneous in the literature and makes it difficult to compare results. The natural history of metastatic cancer is variable, and a general recommendation cannot be made.

In the following articles cure in metastatic cancer of the breast, the liver and GIST are discussed and data 


\section{editorial}

in each tumor type are reviewed by experts in their field.

Conflict of interest H. Kaufmann declares that he has no competing interests.

\section{References}

1. Daneshmand S, Albers P, Fosså SD, et al. Contemporary management of postchemotherapy testis cancer. Eur Urol. 2012;62(5):867-76.

2. Jamison RL, Donohue JH, Nagorney DM, Rosen CB, Harmsen WS, Ilstrup DM. Hepatic resection for metastatic colorectal cancer results in cure for some patients. Arch Surg. 1997;132(5):505-11. https://doi.org/10.1001/ archsurg.1997.
3. Hellman S, Weichselbaum RR. Oligometastases. J Clin Oncol. 1995;13:8-10.

4. Corbin KS, Hellman S, Weichselbaum RR. Extracranial oligometastases: asubsetofmetastases curablewith stereotactic radiotherapy. JClin Oncol. 2013;31:1384-90.

5. TohmeS, Simmons RL, TsungA. Surgery for cancer: a trigger for metastases. Cancer Res. 2017;77(7):1548-52. https:// doi.org/10.1158/0008-5472.CAN-16-1536.

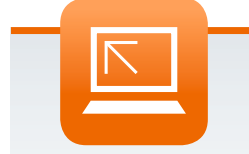

- For latest news from international oncology congresses see: http://www.springermedizin.at/ memo-inoncology 